\title{
Eritema pigmentado fijo secundario a ingesta de alopurinol
}

\section{Fixed erythema pigmentosum secondary to alopurinol ingestion}

\author{
Angélica Villanueva Otamendi, ${ }^{\star}$ Karen Montserrat Ramírez Gómez ${ }^{\ddagger}$
}

\section{RESUMEN}

El eritema fijo pigmentado es una reacción cutánea secundaria a la ingesta de algunos fármacos, del cual aún no se conoce por completo su fisiopatología. Se le asocia principalmente a la administración de antibióticos como la trimetoprima-sulfametoxazol, a antiinflamatorios no esteroideos (AINEs) y psicofármacos. Se caracteriza clínicamente por la presencia de manchas eritematovioláceas que en ocasiones pueden adquirir un aspecto edematoso o con ampollas, que al involucionar dejan una mancha hiperpigmentada de color azul-grisáceo. De manera característica, con la reexposición al fármaco, aparecen en la misma localización. En este artículo se comunica el caso de un paciente del sexo masculino, de 38 años de edad, quien acudió a consulta por presentar manchas eritematovioláceas diseminadas, en quien se realizó el diagnóstico de eritema pigmentado fijo secundario a ingesta de alopurinol.

Palabras clave: Eritema pigmentado fijo, reexposición a fármacos, alopurinol.

\section{ABSTRACT}

Fixed pigmented erythema is a cutaneous reaction secondary to drugs intake, of which pathophysiology it isn't yet fully known. It is mainly associated with antibiotics intake, such as trimethoprim-sulfamethoxazole, NSAIDs, and psychotropic drugs. It's clinically characterized by erythematous-violet plaques that can occasionally appear edematous or with blisters, and resolve to leave a blue-gray hyperpigmented macules. Typically upon drug exposure, they appear in the same location. In this article, we report the case of a 38 years old male patient with disseminated erythematous-violet spots, in who diagnosis of fixed pigmented erythema secondary to allopurinol intake was made.

Keywords: Pigmented fixed erythema, upon drug exposure, allopurinol.

\section{INTRODUCCIÓN}

El espectro de las reacciones cutáneas secundarias a ingesta de fármacos puede manifestarse con erupciones cutáneas comunes leves, o dermatosis muy diseminadas y severas que ponen en peligro la vida del paciente.

Existen diversas presentaciones clínicas de las reacciones cutáneas a fármacos: exantemática, urticariforme, ampollosa o pustulosa. La extensión de las mismas puede ser variable. Una vez que se han analizado la topografía y la morfología de la

\footnotetext{
* Dermatóloga.

‡ Médico residente del tercer año de Dermatología.
}

Centro Dermatológico «Dr. Ladislao de la Pascua», SSCDMX. dermatosis, se puede realizar un diagnóstico clínico específico, por ejemplo: eritema pigmentado fijo, pustulosis exantemática aguda generalizada, etcétera. ${ }^{1}$

El eritema pigmentado fijo (EPF) es una forma de toxicodermia. Fue descrito por primera vez en 1889 por Bourns al observar una erupción en labios y lengua en un paciente que había ingerido 20 gramos de antipirina y que, al curar, dejó una hiperpigmentación residual bien delimitada. ${ }^{2}$ Posteriormente, en 1894, Brocq acuñó el término de «exantema fijo medicamentoso» para describir una erupción eritematopigmentada, fija, secundaria

Citar como: Villanueva OA, Ramírez GKM. Eritema pigmentado fijo secundario a ingesta de alopurinol. Rev Cent Dermatol Pascua. 2021; 30 (1): 20-23. https://dx.doi. org/10.35366/100587 
a la ingesta de antipirina y que tenía la particularidad de reaparecer en la misma localización cutánea unas horas después de readministrar el fármaco desencadenante. ${ }^{3}$ En la literatura internacional actual se manejan los dos términos antes señalados.

EI EPF puede presentarse en personas de cualquier edad, aunque la mayoría de los casos se han reportado en adultos jóvenes. La proporción entre géneros varía según las diferentes series. Tiene una distribución mundial, sin diferencias raciales. ${ }^{3}$

La fisiopatología del EPF no ha sido dilucidada totalmente; sin embargo, al analizar a nivel ultraestructural las lesiones cutáneas, se ha observado la presencia de linfocitos intraepidérmicos - la mayoría del subtipo CD8 (con importante potencial citotóxico) -, pero que mantienen un estado de anergia, sin producir ninguna alteración en los queratinocitos, y permanecen en la piel lesionada durante más de cuatro meses. La reingesta del fármaco responsable del EFP provoca la activación - mediante lgE y otros mecanismos no inmunológicos, como la simple presión física local- de los mastocitos presentes en vasos sanguíneos circundantes, liberando una serie de citocinas como el factor de necrosis tumoral alfa (TNF- $\alpha$ ), que induce a estos queratinocitos a incrementar la expresión de las moléculas de adhesión intercelular ICAM-1, implicadas en la interacción entre queratinocitos y linfocitos, lo cual facilita la activación de los linfocitos CD8 con potencial autolítico. Esto explica la específica localización del EPF.

Clínicamente se caracteriza por la presencia de manchas eritematovioláceas, incluso de color negro, que pueden evolucionar a placas edematosas o ampollosas. Por lo general, son lesiones solitarias, aunque también pueden ser múltiples. Se localizan principalmente en labios, genitales o área perianal, manos y pies; no obstante, pueden ubicarse en cualquier parte de la superficie cutánea. Comúnmente es asintomático, aunque en ocasiones los pacientes refieren sensación de ardor, algunos otros pueden desarrollar fiebre, malestar general o síntomas abdominales. ${ }^{3}$

EI EPF se desarrolla en un lapso que va de 30 minutos hasta ocho o 16 horas después de la ingestión del fármaco. Después de la fase aguda (que puede durar de días a semanas) disminuye la inflamación y persiste una mancha hiperpigmentada residual color azul-gris. ${ }^{1}$

Se describen cinco formas clínicas: la clásica (con placas eritematosas pigmentadas asimétricas), la de placas eritematosas no pigmentadas asimétricas, la forma «minor o frustra», la ampollosa diseminada y la lineal. ${ }^{4}$ Sin embargo, puede manifestarse con reacciones graves, como el síndrome de Stevens-Johnson o el síndrome de necrólisis epidérmica tóxica. En pocos casos también se han descrito foliculitis pustulosa eosinofílica y granuloma anular diseminado. ${ }^{5}$

Tiene origen medicamentoso en $85-100 \%$ de los casos. Los fármacos principalmente involucrados son: antimicrobianos como trimetoprima-sulfametoxazol (principal fármaco involucrado), tetraciclinas, sulfonamida, metronidazol, amoxicilina, ampicilina, clindamicina; antifúngicos; albendazol; AINEs como salicilatos, fenilbutazona, fenacetina, paracetamol y metamizol; psicofármacos como barbitúricos, carbamazepina y benzodiazepinas; otros como quinina, quinidina, dapsona, así como algunos colorantes contenidos en fármacos y alimentos. ${ }^{2,3}$

En pocas publicaciones se ha comunicado el EFP causado por ingesta de alopurinol. ${ }^{6}$ Se manifiesta con lesiones maculopapulares. En 1999 se registró el primer caso de EPF en un paciente de 78 años en tratamiento con alopurinol, quien acudió a consulta por presentar en tórax anterior una placa eritematosa, edematosa, redonda a ovalada, color gris-azul, que se resolvió al descontinuar el fármaco. ${ }^{7}$

El diagnóstico de EPF se realiza con base en la topografía, morfología y el antecedente de aparición de lesiones recurrentes en los mismos sitios al ingerir alguno de los fármacos ya señalados. ${ }^{8}$ El estándar de oro para identificar el agente causal es la prueba de provocación oral; sin embargo, no es recomendable debido a que se puede inducir una reacción grave. ${ }^{9}$ Otros métodos diagnósticos que se han utilizado son las pruebas al parche ${ }^{6}$ y el test de transformación de linfocitos, el cual es seguro y confiable para determinar hipersensibilidad a varios fármacos. ${ }^{10}$

El estudio histopatológico no es necesario para el diagnóstico. Sólo se realiza en los casos dudosos. En la literatura, los hallazgos a este nivel muestran una marcada degeneración hidrópica de la membrana basal, con la presencia de un infiltrado linfocítico a lo largo de la unión dermoepidérmica con necrosis de los queratinocitos, es típica la incontinencia del pigmento. Las vesículas subepidérmicas pueden ser un dato presente en una lesión avanzada; en la dermis superior pueden observarse linfocitos, histiocitos y neutrófilos, los eosinófilos en ocasiones pueden ser prominentes. En las lesiones residuales la incontinencia del pigmento puede ser la única alteración histológica. ${ }^{11}$

Lo más importante para el tratamiento es identificar el fármaco responsable y suspender inmediatamente toda la medicación que sea posible. Localmente, las lesiones no erosionadas pueden mejorar con corticoides tópicos potentes o intralesionales. Es importante valo- 
rar los beneficios y perjuicios que supone para estos pacientes suspender la medicación desencadenante, o bien sustituirla por otra diferente. Se han realizado estudios de desensibilización tras reexposición progresiva al fármaco, llegando a alcanzar dosis terapéuticas sin reaparición de un episodio de EPF. ${ }^{6,12,13}$ A pesar de lo antes mencionado, aún se requieren más estudios de seguimiento.

\section{CASO CLÍNICO}

Paciente del sexo masculino, de 38 años de edad, acude a consulta en el Centro Dermatológico Pascua por presentar desde hace tres meses manchas eritematovioláceas en su cuerpo.

A la exploración física se observa dermatosis diseminada a cabeza, cuello, extremidades superiores e inferiores. De la primera afecta la cara en párpados superiores (Figura 1), y región retroauricular izquierda, del cuello, las caras posterior y lateral derecha (Figura 2); de las extremidades superiores compromete codo derecho y mano izquierda en región dorsal, a nivel de cuarta y quinta articulación metacarpofalángica (Figura 3); y de la extremidad inferior derecha, la pierna en caras anterior y posterior a nivel del tercio medio y pie

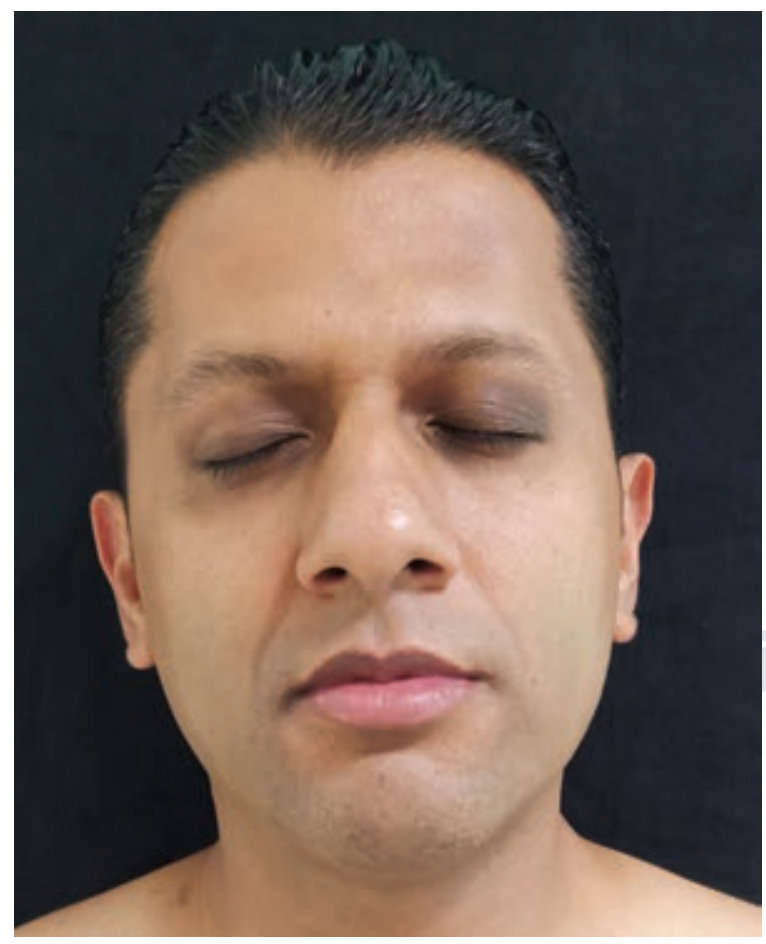

Figura 1: Aspecto clínico de lesión en el párpado izquierdo.

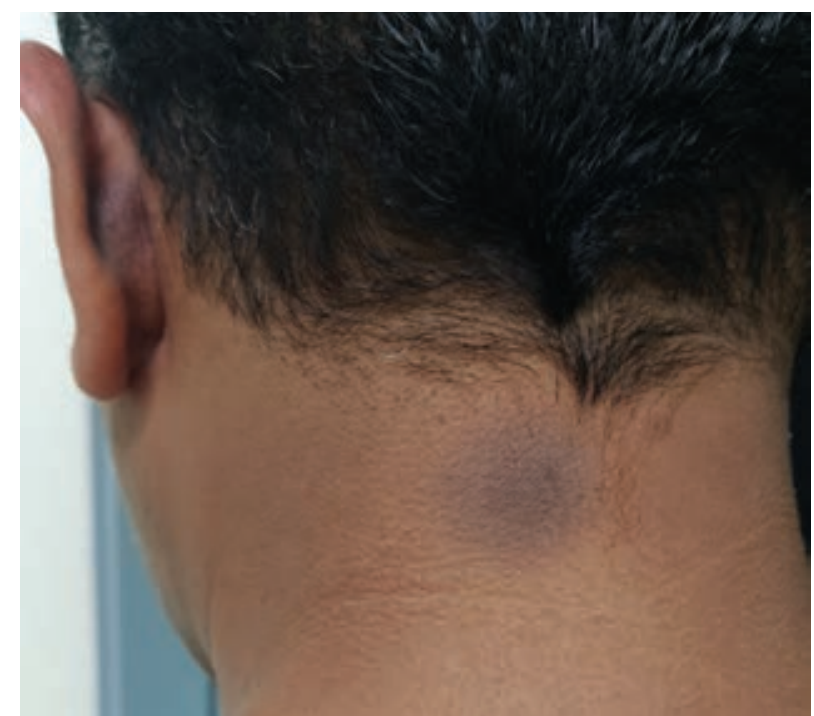

Figura 2: Lesión en región retroauricular izquierda y en cara posterior de cuello.

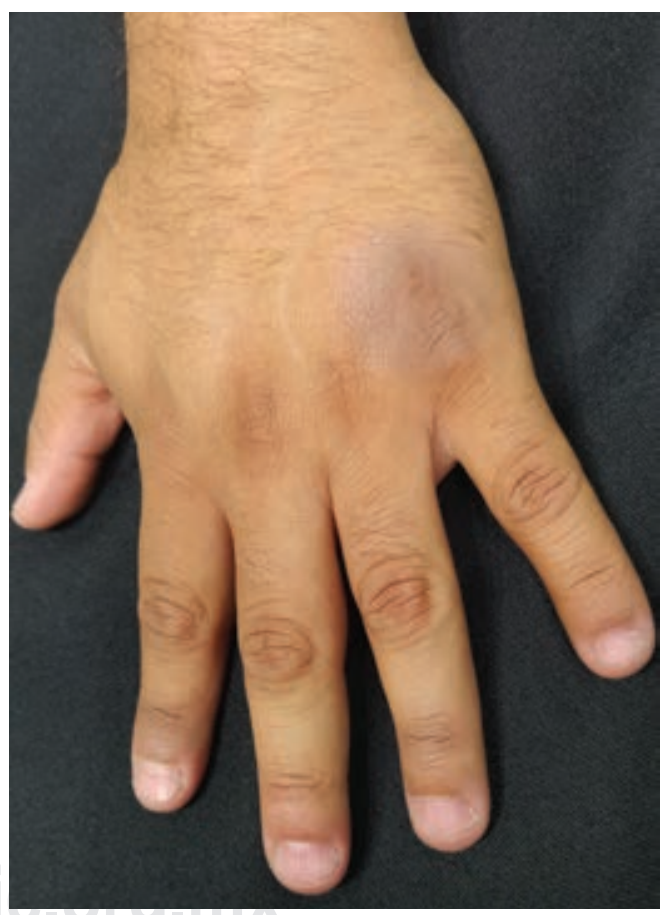

Figura 3: Lesión activa con eritema en dorso de mano izquierda.

en región dorsal (Figura 4 A y $B$ ). La dermatosis es bilateral, asimétrica y está constituida por numerosas manchas hiperpigmentadas color gris-violáceo, una de ellas con discreto eritema, de forma oval, de límites bien definidos, de evolución crónica y asintomática. 

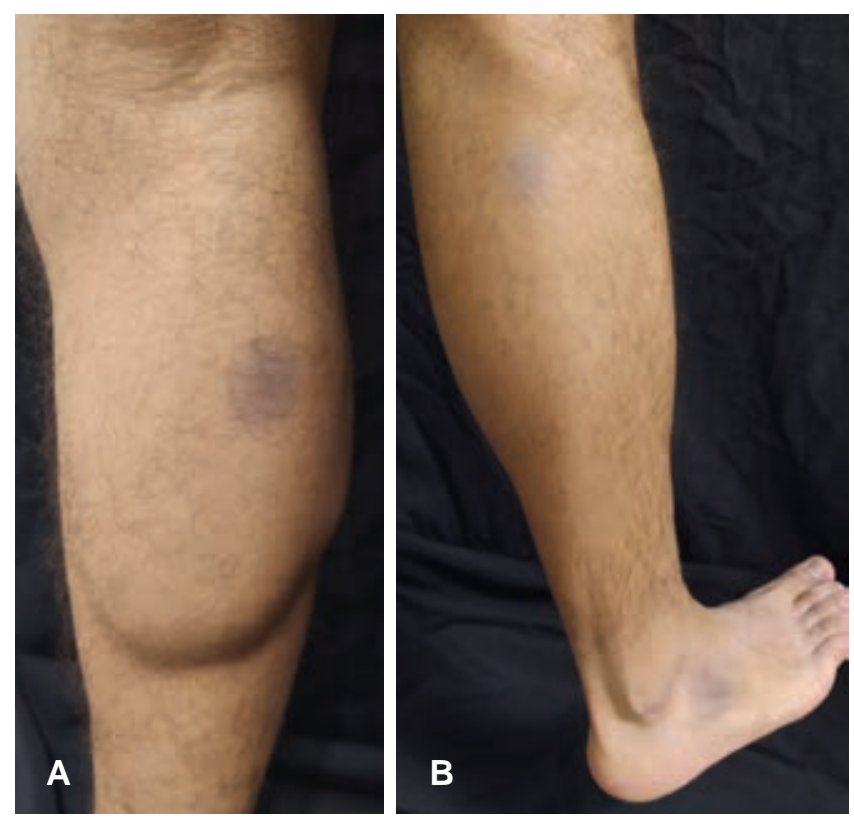

Figura 4: Presencia de lesiones en A) cara posterior de pierna derecha y B) cara externa del pie derecho.

Al interrogatorio dirigido refiere dos ataques previos de gota, agudos, controlados con alopurinol. El último episodio se presentó tres meses antes, momento en el que aparecieron las lesiones. Refiere que en algunos sitios ya habían aparecido manchas desde el primer ataque agudo de gota. Niega la ingesta de algún otro fármaco.

Por la morfología de las lesiones y el antecedente de la ingesta de alopurinol, se realiza el diagnóstico clínico de eritema fijo pigmentado secundario a alopurinol.

Se le indica al paciente medidas generales en la piel, la aplicación de una crema emoliente dos a tres veces al día, así como mometasona ungüento cada 24 horas en lesiones eritematosas por tres semanas, y una crema con urea al $20 \%$ en lesiones pigmentadas cada 12 horas, además de evitar la ingesta de alopurinol. Continúa su seguimiento en el servicio de dermatología general.

\section{COMENTARIO}

La importancia de comunicar este caso radica en que en la literatura internacional existen muy pocos casos semejantes publicados. Hay que tomar en cuenta que el alopurinol es un fármaco utilizado de manera frecuente para el tratamiento de gota y que es prescrito por toda la rama médica. Su baja frecuencia puede ser porque es subdiagnosticado. Es importante que se conozca que el eritema pigmentado fijo es una dermatosis que se puede presentar como efecto secundario a la administración de este fármaco. Por lo tanto, conocer lo anterior facilita a los médicos tratantes realizar un diagnóstico oportuno, así como buscar un tratamiento alternativo para el cuadro de gota, refiriéndolo al servicio de dermatología para valoración y tratamiento de dicha dermatosis.

\section{REFERENCIAS}

1. Fitzpatrick TB. Dermatology in general medicine. Chapter 41 Cutaneous reactions to drugs. New York: McGraw Hill. 2012, pp. 449-450.

2. Muñoz EVF, Valenzuela PGA, Rochín TM. Estudio de 372 casos de eritema fijo pigmentario. Piel. 2011; 26: 213-220.

3. Avilés IJA, Huerta BM, Suárez FR, Lázaro OP. Exantema fijo medicamentoso. Med Integral. 2002; 40: 251-255.

4. Atzori L, Pinna AL, Mantovani L, Ferreli C, Pau M, Mulargia M et al. Cutaneous adverse drug reactions to alopurinol: 10 year observational survey of the dermatology department--Cagliari University (Italy). $J$ Eur Acad Dermatol Venereol. 2012; 26: 1424-1430.

5. Lee HJ, Kim HS, Park YM, Kim HO, Lee JY. Fixed drug eruption due to allopurinol: positive oral provocation. Ann Dermatol. 2011:23: 402-403.

6. Muñoz EVF, Rubio RMZ, Douriet MFA, Domínguez CG. Eritema pigmentado fijo atípico por diaminodifenilsulfona. Bol Med UAS. 2006; 2: 36-39.

7. Tsai TF, Yeh TY. Allopurinol in dermatology. Am J Clin Dermatol. 2010; 11: 225-232.

8. Sehgal VN, Jain S. Allopurinol induced transitory giant fixed drug eruption: an atypical expression. J Dermatol. 1999; 26: 198-199.

9. Muñoz EVF, Valenzuela PGA. Eritema pigmentado fijo genital. Estudio de 69 casos. Rev Cent Dermatol Pascua. 2011; 20: 91-99.

10. Gupta R. Drugs causing fixed drug eruptions: confirmed by provocation test. Indian J Dermatol Venereol Leprol. 2003; 60: 120-121.

11. Kim MH, Shim EJ, Jung JW, Sohn SW, Kang HR. A case of allopurinolinduced fixed drug eruption confirme with a lymphocyte transformation test. Allergy Asthma Immunol Res. 2012; 4: 309-310.

12. Calonje JE, Brenn T, Lazar A, Billings S. McKee's pathology of the skin. Cutaneous adverse reactions to drugs, Chapter 14, 4th ed. Elsevier. 2012, pp. 599-600.

13. Schumacher M. Intravenous desensitization to allopurinol in a heart transplant patient with gout. Ann Allergy Asthma Immunol. 2004; 92: 374-376. 